\title{
SOFOMORE: Combined EEG source and forward model reconstruction
}

\section{Stahlhut, Carsten; Mørup, Morten; Winther, Ole; Hansen, Lars Kai}

Published in:

IEEE International Symposium on Biomedical Imaging: From Nano to Macro, 2009. ISBI '09.

Link to article, DOI:

10.1109/ISBI.2009.5193081

Publication date:

2009

Document Version

Publisher's PDF, also known as Version of record

Link back to DTU Orbit

Citation (APA):

Stahlhut, C., Mørup, M., Winther, O., \& Hansen, L. K. (2009). SOFOMORE: Combined EEG source and forward model reconstruction. In IEEE International Symposium on Biomedical Imaging: From Nano to Macro, 2009. ISBI '09. (pp. 450-453). IEEE. https://doi.org/10.1109/ISBI.2009.5193081

\section{General rights}

Copyright and moral rights for the publications made accessible in the public portal are retained by the authors and/or other copyright owners and it is a condition of accessing publications that users recognise and abide by the legal requirements associated with these rights.

- Users may download and print one copy of any publication from the public portal for the purpose of private study or research.

- You may not further distribute the material or use it for any profit-making activity or commercial gain

- You may freely distribute the URL identifying the publication in the public portal 


\title{
SOFOMORE: COMBINED EEG SOURCE AND FORWARD MODEL RECONSTRUCTION
}

\author{
Carsten Stahlhut, Morten Mørup, Ole Winther, Lars Kai Hansen \\ Technical University of Denmark \\ Department of Informatics and Mathematical Modelling \\ Richard Petersens Plads, 2800 Lyngby, Denmark.
}

\begin{abstract}
We propose a new EEG source localization method that simultaneously performs SOurce and FOrward MOdel REconstruction (SOFOMORE) in a hierarchical Bayesian framework. Reconstruction of the forward model is motivated by the many uncertainties involved in the forward model, including the representation of the cortical surface, conductivity distribution, and electrode positions. We demonstrate in both simulated and real EEG data that reconstruction of the forward model improves localization of the underlying sources.
\end{abstract}

Index Terms - EEG, source reconstruction, uncertain forward model, hierarchical Bayes, distributed model

\section{INTRODUCTION}

Functional neuroimaging by PET and fMRI has added considerably to our understanding of the spatial distribution of information processing in the human brain. To better understand the temporal aspects of systems neuroscience electroand magneto-encephalography (EEG, MEG) are promising modalities due to their high temporal resolution. In addition EEG is attractive as it can be acquired under more naturalistic conditions than PET, fMRI, and MEG. Unfortunately, brain mapping by EEG is an ill-posed problem and relatively strong assumptions are needed for regularization. In the existing literature of source reconstruction significant efforts have been devoted to methods that incorporate spatio-temporal priors to accommodate for focal source distributions under the assumption that the forward model is known. Such inverse methods are typically classified as equivalent current dipole (ECD) or distributed models. ECD models assume that the sources can be described by a small number of ECDs [1], such that the problem is restricted to identify their location and orientation. Distributed models normally assume distributed currents with dipoles oriented perpendicular to the cortical surface. To solve the inverse problem several prior assumptions have been suggested such as mathematical constraints like the (weighted) minimum norm [2] and maximal smoothness [3] properties. Other priors such as anatomical, physiological, and temporal information $[4,5,6]$ have also been proposed. Integrating such prior assumptions into the source reconstruction problem is conveniently implemented in a Bayesian framework. Common to existing source localization methods is that they perform the inversion under the assumption that the forward model is known. However, many noisy processes compromise the forward model, including the representation of the conductivity distribution, the cortical surface, and electrode positions. When 'realistic head models' are constructed from tissue segmentation based on e.g. structural MRI, the geometry of the head model is affected by the resolution and tissue segmentation errors. Such geometric errors can be represented as small magnitude perturbations of the head model shape [7]. Due to the dependence of the head model in the ill-posed EEG source reconstruction problem these uncertainties will also affect the source estimation. Here, we will make a first attempt to correct the uncertain forward model simultaneously with the source localization in order to obtain a more reliable source estimate. Recently, a similar aim was pursued in $[8,9]$, however, from quite a different perspective than ours. In [8] the geometric structure of the forward model is assumed known, while the skull-brain conductivity ratio is an unknown parameter which is estimated simultaneously with the sources. Similar, [9] reported a probabilistic distributed model to account for uncertainty in the skull conductivity. In this contribution, we suggest a more uncommitted approach in which the complete forward model is considered 'uncertain'. Thus, we approach the EEG source localization problem with a hierarchical Bayesian model that simultaneously perform SOurce and FOrward MOdel REconstruction, in short, the SOFOMORE method.

\section{METHODS}

\subsection{The Forward Model}

The relationship between the recorded EEG $\mathbf{M}=\left\{\mathbf{m}_{t}\right\}_{t=1}^{N_{t}}$ from an array of $N_{c}$ sensors placed on the scalp and the neural current activity in the brain is given by

$$
\mathbf{M}=\mathbf{A S}+\mathbf{E},
$$

where $\mathbf{A}$ is the forward model consisting of a set of forward fields $\left\{\mathbf{a}_{i}\right\}_{i=1}^{N_{d}}$ corresponding to each of the dipoles in the primary source current denoted $\mathbf{S}=\left\{\mathbf{s}_{t}\right\}_{t=1}^{N_{t}}$. We assume the 
orientations of current dipoles at the vertices to be perpendicular to the cortical surface and the measurement noise $\mathbf{E}$ is modeled as additive zero-mean Gaussian distributed. The forward model was estimated by the SPM5 academic software (http://www.fil.ion.ucl.ac.uk/spm/), based on routines from BrainStorm (http://neuroimage.usc . edu/brainstorm/) as a basic three-spheres (scalp, skull, brain) head model.

\subsection{SOFOMORE: A Hierarchical Bayes Approach}

Given the linear relationship in Eq. (1) and the Gaussian noise model, for which we will denote the precision by $\beta$, the likelihood, $p(\mathbf{M} \mid \mathbf{S}, \mathbf{A}, \beta)$, can be expressed as a product of multivariate Gaussian distributions $\mathbf{m}_{t} \sim \mathcal{N}\left(\mathbf{A s}_{t}, \beta^{-1} \mathbf{I}_{N_{c}}\right)$. Figure 1 is a graphical representation of the hierarchical model. For the sources we apply a quite similar hierarchical prior as [6], i.e. a zero-mean multivariate Gaussian distribution with a diagonal precision matrix $\mathbf{D}$, with elements $\boldsymbol{\alpha}=\left\{\alpha_{i}\right\}_{i=1}^{N_{d}}$, i.e., $\mathbf{s}_{t} \sim \mathcal{N}\left(\mathbf{0}, \mathbf{D}^{-1}\right)$. In contrast to [6] we model the uncer-

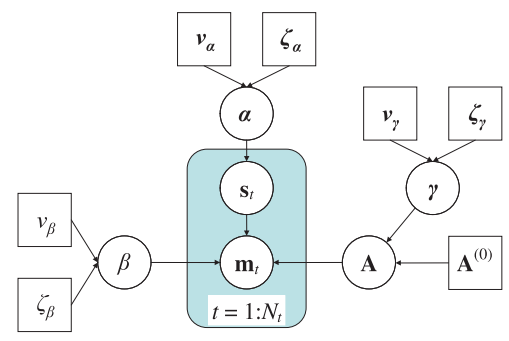

Fig. 1. Graphical representation of hierarchical model that accounts for an uncertain forward model.

tainty of the forward fields, which is performed by independent multivariate Gaussian distributions with prior mean $\mathbf{a}_{i}^{(0)}$ and precision $\gamma_{i}, \mathbf{a}_{i} \sim \mathcal{N}\left(\mathbf{a}_{i}^{(0)}, \gamma_{i}^{-1} \mathbf{I}_{N_{c}}\right)$. The prior mean of the $i^{\text {th }}$ forward field is obtained from the solution to the forward problem. An assignment of a parameter to each of the forward fields allow us to automatically control which of the forward fields that should be corrected if necessary. Hereby, mainly the forward fields for the active sources have to be corrected and the rest remain unchanged if these precisions are large. Conjugate priors for all the precision parameters have been used, with skewness parameter $\nu_{x}$ and inverse scale parameter $\zeta_{x}$, see Fig.1, i.e. precision parameter $x$ is modeled with a Gamma distribution $\mathcal{G}\left(x \mid \nu_{x}, \zeta_{x}\right)$.

\subsubsection{Variational Bayesian Formulation}

The Bayesian framework provides the complete joint posterior of sources and parameters given the observed data $\mathbf{M}$. As a representation of the sources we use the marginal posterior distribution

$$
p(\mathbf{S} \mid \mathbf{M})=\frac{p(\mathbf{M}, \mathbf{S})}{p(\mathbf{M})}=\frac{\int p(\mathbf{M}, \boldsymbol{\theta}) \mathrm{d} \boldsymbol{\theta}_{\backslash \mathbf{S}}}{p(\mathbf{M})},
$$

Table 1. VB updates for hierarchical model with $\langle\cdot\rangle$ denoting the expectation and $\mathbf{l}_{j}$ the $j^{\text {th }}$ lead field i.e. $j^{\text {th }}$ row in $\mathbf{A} . \mathbf{G}$ is a diagonal matrix with $\boldsymbol{\lambda}$ in the diagonal, $\psi(\cdot)$ is the digamma function and its derivative is $\psi^{\prime}(\cdot)$. Hyperhyperparameters of the form $\nu_{x}$ and $\zeta_{x}$ are updated using Newton-Raphson.

\begin{tabular}{ll}
\hline$q(\boldsymbol{\theta})$ & VB updates \\
\hline $\mathcal{N}\left(\mathbf{s}_{t} \mid \boldsymbol{\mu}_{t}, \mathbf{\Sigma}\right)$ & $\boldsymbol{\mu}_{t}=\mathbf{\Sigma}\langle\mathbf{A}\rangle^{T} \mathbf{m}_{t}\langle\beta\rangle, \boldsymbol{\Sigma}=\left(\left\langle\beta \mathbf{A}^{T} \mathbf{A}\right\rangle+\langle\mathbf{D}\rangle\right)^{-1}$ \\
$\mathcal{N}\left(\mathbf{l}_{j} \mid \boldsymbol{\eta}_{j}, \boldsymbol{\Psi}\right)$ & $\boldsymbol{\eta}_{j}=\boldsymbol{\Psi}\left(\langle\mathbf{G}\rangle \mathbf{l}_{j}^{(0)}+\langle\beta\rangle \sum_{t=1}^{N_{t}}\left\langle\mathbf{s}_{t}\right\rangle m_{j t}\right)$ \\
& $\boldsymbol{\Psi}=\left(\langle\mathbf{G}\rangle+\langle\beta\rangle \sum_{t=1}^{N_{t}}\left\langle\mathbf{s}_{t} \mathbf{s}_{t}^{T}\right\rangle\right)^{-1}$ \\
$\mathcal{G}\left(\alpha_{i} \mid \widehat{\nu}_{\alpha_{i}}, \widehat{\zeta}_{\alpha_{i}}\right)$ & $\widehat{\nu}_{\alpha_{i}}=\nu_{\alpha_{i}}+\frac{N_{t}}{2}, \widehat{\zeta}_{\alpha_{i}}=\zeta_{\alpha_{i}}+\frac{1}{2} \sum_{t=1}^{N_{t}}\left\langle s_{i t}^{2}\right\rangle$ \\
$\mathcal{G}\left(\gamma_{i} \mid \widehat{\nu}_{\gamma_{i}}, \widehat{\zeta}_{\gamma_{i}}\right)$ & $\widehat{\nu}_{\gamma_{i}}=\nu_{\gamma_{i}}+\frac{N_{c}}{2}, \widehat{\zeta}_{\gamma_{i}}=\zeta_{\gamma_{i}}+\frac{1}{2}\left\langle\left(\mathbf{a}_{i}-\mathbf{a}_{i}^{(0)}\right)^{2}\right\rangle$ \\
$\mathcal{G}\left(\beta \mid \widehat{\nu}_{\beta}, \widehat{\zeta}_{\beta}\right)$ & $\widehat{\nu}_{\beta}=\nu_{\beta}+\frac{N_{c} N_{t}}{2}, \widehat{\zeta}_{\beta}=\zeta_{\beta}+\frac{1}{2} \sum_{t=1}^{N_{t}}\left\langle\left(\mathbf{m}_{t}-\mathbf{A s}_{t}\right)^{2}\right\rangle$ \\
\hline & $\nu_{x}^{(k+1)}=\nu_{x}^{(k)} \exp \left\{-\frac{\psi\left(\nu_{x}^{(k)}\right)-\ln \nu_{x}^{(k)}+\ln \langle x\rangle-\langle\ln x\rangle}{\nu_{x}^{(k)} \psi^{\prime}\left(\nu_{x}^{(k)}\right)-1}\right\}$ \\
\hline & $\zeta_{x}=\frac{\nu_{x}}{\langle x\rangle}$
\end{tabular}

where $\boldsymbol{\theta}=\{\mathbf{S}, \mathbf{A}, \boldsymbol{\alpha}, \beta, \gamma\}, \boldsymbol{\theta} \backslash \mathbf{S}$ denotes parameter set $\boldsymbol{\theta}$ except for $\mathbf{S}, p(\mathbf{M}, \boldsymbol{\theta})$ is the joint distribution, and $p(\mathbf{M})$ is the marginal likelihood. Due to the hierarchical structure of the model, the marginal likelihood becomes analytically intractable. Thus, approximations are needed and we will apply a standard Variational Bayesian (VB) framework [10], in which the joint posterior $p(\boldsymbol{\theta} \mid \mathbf{M})$ is approximated by a parameterized simpler distribution $q(\boldsymbol{\theta})$ whose parameters are determined by maximizing the bound on the marginal likelihood obtained through Jensen's inequality,

$$
\mathcal{L}=\ln \int p(\mathbf{M}, \boldsymbol{\theta}) \mathrm{d} \boldsymbol{\theta} \geq \int q(\boldsymbol{\theta}) \ln \frac{p(\mathbf{M}, \boldsymbol{\theta})}{q(\boldsymbol{\theta})} \mathrm{d} \boldsymbol{\theta} .
$$

The approximate posterior is taken as fully factored in the parameters $\boldsymbol{\theta}=\{\mathbf{S}, \mathbf{A}, \boldsymbol{\alpha}, \beta, \gamma\}$. The resulting sequential $\mathrm{VB}$ updates are given in Tab. ${ }^{1}$.

\section{EXPERIMENTS}

We demonstrate the viability of the SOFOMORE approach on simulated data and real EEG data. We will show that estimation of the forward model matters, by comparing reconstructed sources with the corresponding model with fixed forward model. The results in this section are for clarity obtained by operating directly on the measurements $\mathbf{M}$ without preprocessing of data like bandpass filtering or use of temporalsubspaces. Extensions to include this can be performed and

\footnotetext{
${ }^{1}$ Note that inversion of covariance matrices of size $N_{d} \times N_{d}$ appears in the VB update equations, which in the conventional approach can be performed efficiently with the matrix inversion lemma when $\mathbf{A}$ is assumed known. However, due to the inter-dependence of $\mathbf{S}$ and $\mathbf{A}$ the matrix inversion lemma cannot be used efficiently. Thus, in the implementation we have performed the VB updates in a subspace spanned by the basis of the lead fields (rows in $\mathbf{A}$ ).
} 
will in general improve performance, future work will concern this important issue.

\subsection{Simulations}

In the simulations we construct a source signal of a half sine of duration $50 \mathrm{~ms}$ with a starting time at $t=25 \mathrm{~ms}$ for a small set of sources located in the occipital lope, as illustrated in the SPM glass-brain representation in Fig.2(a). Note that minor activity seems to appear at the inner part of the left hemisphere, which is due to the mapping from cortex to the glass-brain representation. The simulated clean EEG was constructed with forward model consisting of 3-spheres with tissue conductivities brain:skull:scalp $=0.33: 0.0041: 0.33 \mathrm{~S} / \mathrm{m}$ (ratio 1:1/80:1) and dimension $128 \times 7204$. We corrupted the clean EEG with 'realistic EEG noise' obtained from the pre-stimulus period in an evoked EEG study described in Sec. 3.2. We applied a signal-to-noise ratio $\mathrm{SNR}=10$, with SNR defined as SNR $=P_{\text {cEEG }} / P_{\text {noise }}$, where $P_{\text {cEEG }}$ and $P_{\text {noise }}$ are the power of the clean EEG and noise, respectively. Conductivity errors were added to one of the forward models used in the source localization. In the source reconstruction we used a cortical resolution of 4004 vertices, such that the 'true' forward model has a higher spatial resolution. Figures

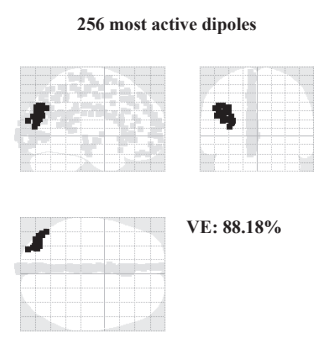

(a) True sources

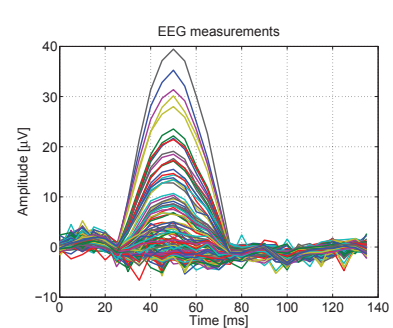

(b) EEG with noise
Fig. 2. Simulated source density and EEG. VE: Variance explained.

3(a) and 3(b) show the estimated source densities at the time $t=50 \mathrm{~ms}$, which corresponds to the time point where we have the maximum amplitude of the EEG. In these figures no corrections of the forward fields are performed but different conductivities have been used. In both cases erroneous activity is estimated in the right pre-motor area (indicated with a circle in the figure). However, a 'correct' choice of conductivities also leads to quite good localization of the true sources. In contrast an erroneous choice of conductivities in Fig.3(a) results in poorer reconstruction of the true sources. The poorer reconstruction can also be seen from a lower log-evidence value and an increase in the variance explained (VE) compared to the correct one in Fig.2(a). The percentages of variance explained in both models are larger than the 'true' value (88.2\%), indicating some overfitting. In Figs.3(c)-(d) we use the same forward models as in Figs.3(a)- (b) as prior means $\left(\mathbf{A}^{(0)}\right)$ in the SOFOMORE model. Indeed the combined Bayesian estimation of forward model and source density leads to a better estimation of the true source density in both cases. Moreover, the resulting estimates are quite similar. However, the model with 'correct' prior mean explains $91.7 \%$ of the data variance, which is closer to the true value than the one with the erroneous forward model taken as prior mean. Additionally, the model in Fig. 3(d) has a much larger log-evidence value, indicating a more likely model.

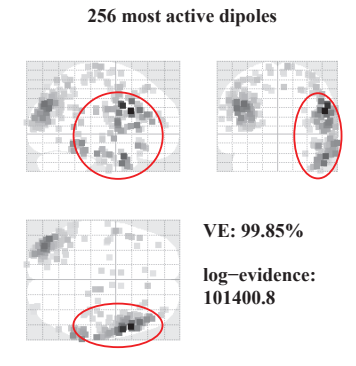

(a) Ratios 1:1/15:1

256 most active dipoles

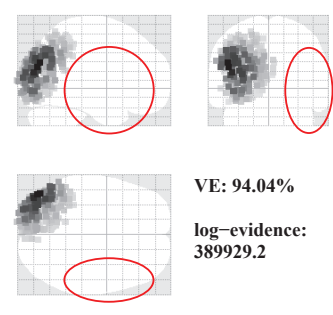

(c) Init ratios $1: 1 / 15: 1$

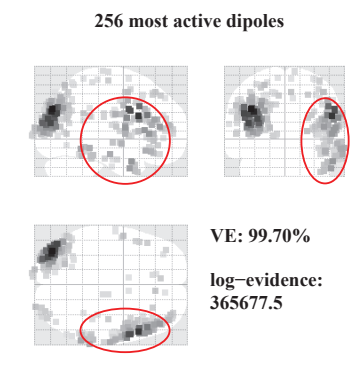

(b) Ratios 1:1/80:1

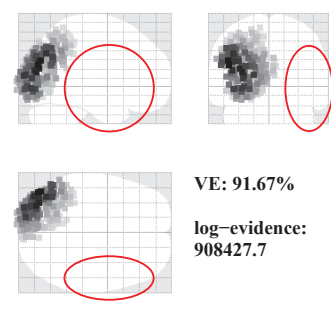

(d) Init ratios 1:1/80:1

Fig. 3. Estimated activity at $t=50 \mathrm{~ms}$ and weighted by the square root of the inverse variance $\boldsymbol{\alpha}$ (i.e. $s_{i t} \sqrt{\alpha_{i}}$ ). First row: No corrections of forward fields. Second row: Corrections are integrated into the source localization method. Different conductivity ratios are used. Figs.(a)+(b) misleading activity inside circles - compare with Fig.2(a). VE: Variance explained.

\subsection{Real EEG data}

This EEG data set is part of a multi-modal study on face perception, where faces and scrambled faces were presented for $600 \mathrm{~ms}$ every $3600 \mathrm{~ms}$ to a subject. A detailed description of the experiment is given in [11] and http://www . fil.ion.ucl.ac.uk/spm (where the data is available for download). In this contribution we reconstruct the average event related potential (ERP) of trials involving real faces as stimuli. In Figs. 4(a) and 4(b) the estimated activity is illustrated at $t=170 \mathrm{~ms}$ after stimulus, without and with integration of the reconstruction of the forward fields respectively. When there is no correction of the forward fields the reconstruction results in quite scattered activity, with a prominent 
voxel located in the right temporal lope and less prominent voxels in the right occipital region. In contrast the SOFOMORE model leads to improved localization of activity in the visual cortex with minor activity in the fusiform gyrus, which is known to be connected with face processing. This result is well-aligned with results reported in [12]. Please note that no spatial smoothing has been performed in our results, which would probably give better localized sources for the case without corrections of $\mathbf{A}$. Moreover, the columns of the forward matrix can be normalized such that the enhancement of the superficial sources is reduced.

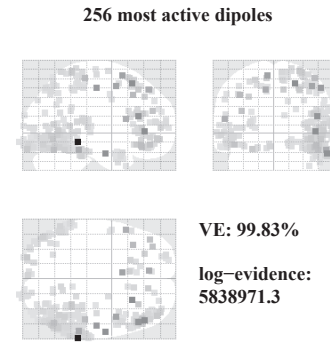

(a) No corrections of $\mathbf{A}$

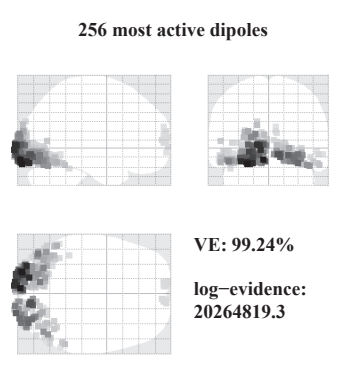

(b) Corrections of $\mathbf{A}$
Fig. 4. Estimated activity $t=170 \mathrm{~ms}$ after stimulus and weighted by the square root of the inverse variance $\boldsymbol{\alpha}$ when no corrections of forward fields are performed and when corrections are integrated into the source localization method. Tissue conductivities brain:skull:scalp $=0.33: 0.0041: 0.33 \mathrm{~S} / \mathrm{m}$ are used. VE: Variance explained.

\section{CONCLUSION}

We proposed a hierarchical Bayesian framework for simultaneous source and forward model reconstruction aimed at a improved source density estimate. Results from simulations and real EEG data illustrated the applicability of the model. We found that the combined reconstruction resulted in more localized activity with fewer large distance errors, in comparison with a similar model with a fixed forward model. Overfitting is a serious concern in the extended model. By invoking a flexible prior on the forward model corrections relative to the prior mean - the standard forward model - are only made where it is necessary, hence reducing overfit. Future work concerns the performance of the SOFOMORE model on more realistic head models based on boundary element method and finite element method and more realistic priors with temporal structure.

\section{REFERENCES}

[1] J. C. Mosher, P. S. Lewis, and R. M. Leahy, "Multiple dipole modeling and localization from spatio-temporal meg data," IEEE Trans. on Biomed. Eng., vol. 39, no. 6, pp. 541-557, 1992.

[2] M.S. Hämäläinen and R.J. Ilmoniemi, "Interpreting magnetic fields of the brain: minimum norm estimates," Med. Biol. Eng. Comput., vol. 32, pp. 35-42, 1994.

[3] R. D. Pascual-Marqui, C. M. Michel, and D. Lehmann, "Low resolution electromagnetic tomography: a new method for localizing electrical activity in the brain," Int J. Psychophysiol., vol. 18, pp. 49-65, 1994.

[4] S. Baillet and L. Garnero, "A bayesian approach to introducing anatomo-functional priors in the eeg/meg inverse problem," IEEE Trans. on Biomed. Eng., vol. 44, no. 5, pp. 374-385, 1997.

[5] C. Phillips, M.D. Rugg, and K.J. Friston, "Anatomically Informed Basis Functions for EEG Source Localisation: Combining Functional and Anatomical Constraints," NeuroImage, vol. 16, no. 3, pp. 678-695, 2002.

[6] M.-A. Sato, T. Yoshioka, S. Kajihara, K. Toyama, N. Goda, K. Doya, and M. Kawato, "Hierarchical bayesian estimation for MEG inverse problem.," Neuroimage, vol. 23, no. 3, pp. 806-26, November 2004.

[7] N. von Ellenrieder, C.H. Muravchik, and A. Nehorai, "Effects of geometric head model perturbation on the EEG forward and inverse problems," IEEE Trans. Biomed. Eng., vol. 53, no. 3, pp. 421-429, March 2006.

[8] S. Lew, C. Wolters, A. Anwander, S. Makeig, and R.S. MacLeod, "Low resolution conductivity estimation to improve source localization," in New Frontiers in Biomag. Proceedings of the 15th Int. Conf. on Biomag., 2007, vol. 1300 of Int. Congress Series, pp. 149-152.

[9] S.M. Plis, J.S. George, S.C. Jun, D.M. Ranken, P.L. Volegov, and D.M. Schmidt, "Probabilistic forward model for electroencephalography source analysis," Physics in Medicine and Biology, vol. 52, no. 17, pp. 5309-5328, 2007.

[10] C.M. Bishop, Pattern Recognition and Machine Learning, Springer, NY 10013 (USA), 2006.

[11] R.N.A. Henson, Y. Goshen-Gottstein, T. Ganel, L.J. Otten, A. Quayle, and M.D. Rugg, "Electrophysiological and hemodynamic correlates of face perception, recognition and priming," Cerebral Cortex, vol. 13, pp. 793 805, 2003.

[12] K. Friston, L. Harrison, J. Daunizeau, S. Kiebel, C. Phillips, N. Trujillo-Barreto, R. Henson, G. Flandin, and J. Mattout, "Multiple sparse priors for the M/EEG inverse problem," NeuroImage, vol. 39, pp. 1104-1120, 2008. 